\title{
Influence of Pre-Existing Mild Cognitive Impairment and Dementia on Post-Stroke Mortality. The Dijon Stroke Registry
}

\author{
Mathilde Graber Lucie Garnier Sophie Mohr Benoit Delpont \\ Christelle Blanc-Labarre Catherine Vergely Maurice Giroud Yannick Béjot \\ Dijon Stroke Registry, EA7460, Pathophysiology and Epidemiology of Cerebro-Cardiovascular Diseases (PEC2), \\ University Hospital of Dijon, University of Burgundy, Dijon, France
}

\section{Keywords}

Stroke $\cdot$ Registry $\cdot$ Epidemiology $\cdot$ Mild cognitive impairment · Dementia · Mortality · Case-fatality

\begin{abstract}
Objective: We assessed the association between pre-stroke cognitive status and 90-day case-fatality. Methods: Patients with ischemic stroke (IS) or spontaneous intracerebral hemorrhage (ICH) were prospectively identified among residents of Dijon, France, between 2013 and 2015, using a population-based registry. Association between pre-stroke cognitive status and case-fatality at 90 days was evaluated using Cox regression. Results: Seven hundred sixty-two patients were identified, and information about pre-stroke cognitive status was obtained for 716 (92.6\%) of them, including 603 IS (84.2\%) and 113 ICH (15.8\%). Before stroke, 99 (13.8\%) patients had mild cognitive impairment (MCl) and 98 (13.7\%) had dementia. Patients with cognitive impairment were older, had a higher prevalence of several risk factors, more severe stroke, more frequent $\mathrm{ICH}$, and less admission to stroke unit. Case-fatality rate at 90 days was $11.7 \%$ in patients without cognitive impairment, $32.3 \%$ in $\mathrm{MCl}$ patients, and $55.1 \%$
\end{abstract}

in patients with dementia. In multivariable analyses, pre-existing $\mathrm{MCl}$ (hazard ratio [HR] 2.22, 95\% Cl 1.21-4.05, $p=0.009$ ) and dementia (HR 4.35, 95\% Cl 2.49-7.61, $p<0.001$ ) were both associated with 90-day case-fatality. Conclusion: Prestroke $\mathrm{MCl}$ and dementia were both associated with increased mortality. These associations were not fully explained by baseline characteristics, pre-stroke dependency, stroke severity or patient management, and underlying reasons need to be investigated.

( 2019 S. Karger AG, Basel

\section{Introduction}

Although the incidence of stroke in high-income countries has stabilized or decreased over the last 2 decades $[1,2]$, the aging population has been associated with an increase in the absolute annual number of cases, especially in elderly people $[2,3]$. Currently, about $60 \%$ of stroke patients are $>75$ years old, and $40 \%$ are $>80$ years

This paper is part of the Dijon Stroke Registry Series.

$\begin{aligned} & \text { karger@karger.com } \\ & \text { www.karger.com/ned }\end{aligned}$
Karger ${ }^{\prime /}$

Prof. Yannick Béjot

Department of Neurology, Dijon Stroke Registry University Hospital of Dijon, Hôpital François Mitterrand 14 rue Paul Gaffarel, BP 77908, FR-21079 Dijon Cedex (France)

E-Mail ybejot@yahoo.fr 
old, and these proportions are expected to rise in the coming years according to demographic estimates [3, 4]. As a consequence, stroke physicians find themselves treating more and more elderly patients in their clinical practice, and, since the epidemiology of cognitive disorders is greatly associated with age [5], a significant proportion of these patients have cognitive impairment prior to stroke onset [6].

In this context, several studies have suggested that demented patients may have a poorer prognosis after stroke [7-9]. More frequent acute complications, less access to a dedicated stroke care unit, and fewer diagnostic resources or acute treatments are some of the reasons put forth to explain these findings [9-11]. However, dementia is the extreme end of cognitive disorders, and whether mild cognitive impairment (MCI) may affect outcome after stroke has been far less evaluated.

Therefore, in this population-based study, we aimed to assess the association between pre-stroke cognitive impairment, including both MCI and dementia, and mortality after stroke.

\section{Methods}

\section{Study Population and Case-Ascertainment Procedures}

This study was based on epidemiological data dated January 1 , 2013 to December 31,2015 prospectively collected from the Dijon Stroke Registry. This is an ongoing population-based study that complies with the defined criteria for conducting "ideal" incidence stroke studies [12], and the guidelines for the reporting of incidence and prevalence studies in neuroepidemiology according to Standards of Reporting of Neurological Disorders [13]. Case-collection and adjudication procedures have been described extensively elsewhere [14]. Briefly, multiple overlapping sources of information are used to identify all cases of stroke and transient ischemic attack (TIA) that occur among residents of the city of Dijon, France (155,000 inhabitants) using "hot" and "cold" pursuit procedures. These procedures include: a review of medical records of all patients referred to the Dijon University Hospital where the only stroke unit is located, as well as a review of computerized hospital diagnostic codes using the International Classification of Diseases, tenth revision (I61, I62, I63, I64, G45, G46, and G81); a review of medical records from the departments of the private hospitals of the city and its suburbs; cooperation with local general practitioners and private neurologists to identify stroke patients from home or nursing homes, and Dijon residents who had a stroke when outside the city; a review of the medical records of patients identified from a computer-generated list of all requests for imaging to radiology centers in Dijon; and regular reviewing of death certificates to identify fatal strokes that occurred outside the hospital. Senior neurologists trained in stroke ascertainment are responsible for final stroke adjudication according to the WHO diagnostic criteria (i.e., "rapidly developing clinical signs of focal, at time global, disturbance of cerebral func- tion, lasting more than $24 \mathrm{~h}$ or leading to death with no apparent cause other than that of vascular origin") [15]. We only included patients with ischemic stroke (IS) and spontaneous intracerebral hemorrhage (ICH).

\section{Data Collected and Outcome}

At registration, the following vascular risk factors and past medical history were collected [14]: hypertension (high blood pressure recorded in a patient's medical history or patients under antihypertensive treatment), diabetes mellitus (glucose level $\geq 7.8 \mathrm{mmol} / \mathrm{L}$ reported in the medical record or patients taking insulin or oral hypoglycemic agents), hypercholesterolemia (total cholesterol level $\geq 5.7 \mathrm{mmol} / \mathrm{L}$ reported in the medical history or patients treated with lipid-lowering therapy), smoking status (current smoker, past smoker or never smoker), history of coronary heart disease, history of stroke or TIA, peripheral artery disease, heart failure, excessive alcohol consumption (defined as alcohol intake $\geq 3$ units a day in men and $\geq 2$ in women), active cancer, and atrial fibrillation (either previously known or diagnosed during stroke evaluation). Premorbid therapies including oral anticoagulants, antiplatelet agents, antihypertensive treatment, and statins were recorded.

The functional status before stroke was evaluated using premorbid modified Rankin Scale score. Pre-stroke cognitive status was assessed based on interviews with patients, their relatives or their general practitioner, as well as the review of medical files. Overlapping sources of information were analyzed by investigators including primary care record, individual consultation records, all hospital outpatient clinic and hospitalization letters obtained from a unique electronic medical file. The only cognitive impairment confirmed by a doctor based on the clinical evaluation before stroke was considered, and final adjudication was finally made by an investigator of the Dijon Stroke Registry based on the overall information collected. Patients were classified as follows: no cognitive impairment, MCI defined as a cognitive decline without any interference with activities of daily life, or dementia defined as a cognitive decline sufficient to interfere with independence in activities of daily living or patients receiving specific treatment for Alzheimer's disease. Patients in whom it was impossible to obtain reliable information were considered as having missing data about pre-stroke cognitive impairment. Stroke severity at onset was quantified by the means of the National Institutes of Health Stroke Scale (NIHSS) score obtained at the first clinical examination. In $29 \%$ of cases, the NIHSS score was estimated retrospectively on the basis of medical records and charts, as previously validated in the literature [16]. For hospitalized patients, medical complications occurring during the acute portion of the stay were collected.

The outcome measured was all-cause case-fatality at 90 days. Vital status was systematically recorded, thanks to the use of death certificates. Information was available for all patients.

\section{Statistical Analyses}

Proportions and mean values of baseline characteristics were compared using the chi-square test, the Wilcoxon-Mann-Whitney test, and the Kruskal-Wallis test when appropriate. Person-days were calculated from the date of stroke at onset until death, the last contact date, and the end of follow-up at 90 days. Survival curves of patients according to their cognitive status before stroke were obtained using Kaplan-Meier analysis. Cox regression models 
were used to evaluate the association between premorbid cognitive status and death at 90 days. The proportional-hazards assumption was checked. In multivariable analyses, we first introduced age and gender. Then, baseline variables (medical history, stroke type, and NIHSS score) with a $p$ value $<0.20$ in unadjusted models were introduced in a second model. Statistical interaction terms between pre-stroke cognitive status and the variables included in the final model were tested using the likelihood-ratio test. Additional analyses were performed on hospitalized patients only. In the multivariable model, admission to the intensive care stroke unit was added as a confounding variable. Finally, to account for acute stroke treatment (IV thrombolysis and mechanical thrombectomy), a subgroup analysis restricted to IS patients was also performed. Values of $p<0.05$ were considered statistically significant. Statistical analysis was performed with STATA@13 software (StataCorp LP, College Station, TX, USA).

\section{Ethics}

The Dijon Stroke Registry was approved by the Comité d'Evaluation des Registres (French National Committee of Registers), Santé Publique France (French Institute for Public Health Surveillance), and the Commission Nationale Informatique et Liberté (French data protection authority).

\section{Results}

Over the study period, 762 patients with acute stroke were recorded. Information about cognitive status prior to stroke was available in 716 (94\%) patients, including 603 IS (84.2\%) and $113 \mathrm{ICH}$ (15.8\%) patients. Among these patients, 99 (13.8\%) had pre-existing MCI and 98 (13.7\%) pre-existing dementia.

Baseline patient characteristics are given in Table 1. Several differences were observed according to cognitive status. Older age at onset, as well as a greater prevalence of atrial fibrillation, history of stroke, heart failure, and premorbid use of anticoagulants and antihypertensive therapy, were noted in patients with MCI or dementia. Stroke subtype distribution also differed between groups; there was a greater proportion of ICH in MCI patients (20.2\%) and patients with dementia (24.5\%) than in patients without cognitive impairment (13.3\%). Additionally, a greater severity at onset was observed in patients with MCI and even more so in patients with dementia. Patients with dementia were more likely to be living in an institution than MCI patients or those without cognitive impairment. Moreover, they were less likely to be hospitalized for acute stroke management ( 79.6 vs. $97 \%$ of MCI patients and $96.2 \%$ of patients without cognitive impairment). When considering hospitalized patients, admission to a stroke unit was more frequent in patients without cognitive im- pairment $(56.3 \%)$ than in those with MCI $(43.8 \%)$ or dementia (29.5\%). Among IS patients, those with dementia were less likely to receive IV thrombolysis. No patient with MCI or dementia was treated with mechanical thrombectomy.

At 90 days, 172 patients had died (22.6\%) in the overall cohort. The case-fatality rate was $11.7 \%$ (95\% CI 9.214.8 ) in patients without cognitive impairment, $32.3 \%$ (95\% CI23.7-42.3) in MCI patients, and 55.1\% (95\% CI 45.0-64.8) in patients with dementia (Fig. 1). In univariate analyses, both MCI (hazard ratio [HR] 3.15, 95\% CI 2.06-4.84, $p<0.001$ ), and dementia (HR 6.16, 95\% CI $4.26-8.89, p<0.001)$ were associated with increased 90 day mortality when compared with patients without cognitive impairment. After adjustment for risk factors and clinical variables, the associations remained significant in multivariable models (adjusted HR 1.90, 95\% CI 1.133.17, $p=0.014$ for MCI and adjusted HR 3.14, 95\% CI $1.96-5.05, p<0.001$ for dementia; Table 2). Further adjustment on pre-stroke dependency (modified Rankin Scale $\geq 2$ ) did not alter the results (adjusted HR 2.22, 95\% CI 1.21-4.05, $p=0.009$ for MCI and adjusted HR 4.35, $95 \%$ CI 2.49-7.61, $p<0.001$ for dementia). No significant statistical interaction was found for each of the variables included in the models.

Analyses restricted to hospitalized patients $(n=673$; $94 \%$ of patients with available data about cognitive status) revealed a similar excess risk of death in patients with MCI (adjusted HR 2.03, 95\% CI 1.22-3.40, $p=0.007$ ) and dementia (adjusted HR 3.35, 95\% CI 2.04-5.49, $p<0.001)$ after adjustment for clinical variables and risk factors (Table 3). The associations remained significant after adjustment for hospitalization in a stroke care unit (adjusted HR 1.91, 95\% CI 1.13-3.21, $p=0.015$ for MCI and adjusted HR 2.97, 95\% CI 1.79-4.91, $p<0.001$ for dementia), even after adjustment for pre-stroke dependency (adjusted HR 2.25, 95\% CI 1.22-4.16, $p=0.01$ for MCI and adjusted HR 4.08, 95\% CI 2.24-7.41, $p<0.001$ for dementia). In a subgroup analysis restricted to IS patients, after adjustment for confounding variables including IV thrombolysis and mechanical thrombectomy, dementia was associated with an increased risk of death (adjusted HR 2.28, 95\% CI 1.23-4.23, $p=0.009$ ), whereas the association was no longer significant for MCI (adjusted HR 1.54, 95\% CI 0.82-2.89, $p=0.18$ ).

To further explore these results, we analyzed the medical complications that occurred during the acute stay of hospitalized patients (Table 4). The only difference found was a greater prevalence of acute confusion in MCI patients (16.7\%) and patients with dementia 
Table 1. Baseline characteristics of stroke patients according to pre-stroke cognitive status

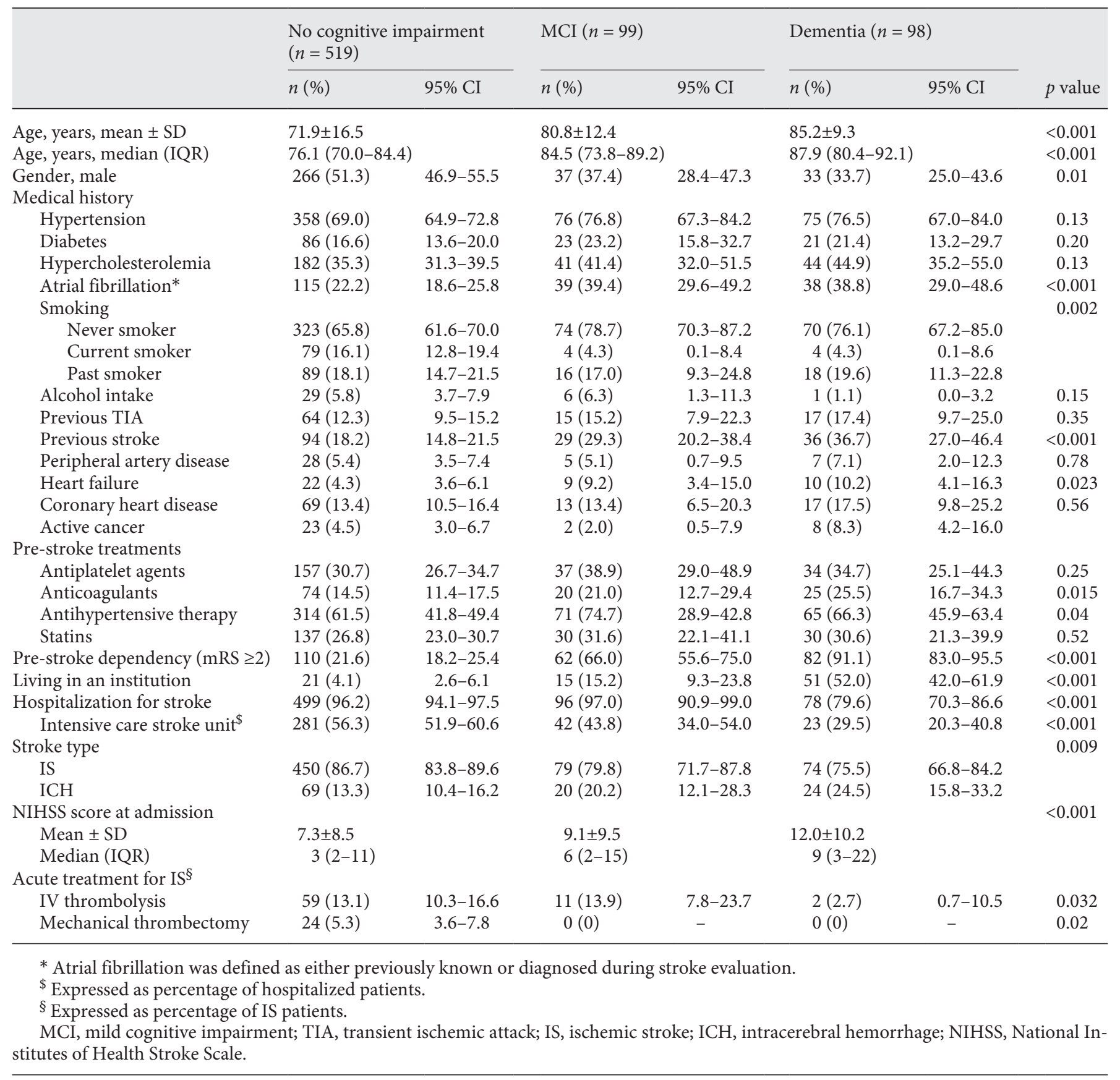

(10.3\%), as compared with patients without cognitive impairment $(2.8 \%)$. The addition of confusion to the multivariable Cox model did not alter our results, and both MCI (adjusted HR 1.86, 95\% CI 1.10-3.14, $p=$ 0.021 ) and dementia (adjusted HR 2.98, 95\% CI $1.81-4.91, p<0.001)$ were still associated with 90 -day case fatality.

Prior Cognitive Status and Post-Stroke Mortality

\section{Discussion}

The present study found that about $14 \%$ of stroke patients had pre-existing MCI and $14 \%$ had pre-existing dementia. Compared with patients whose medical history showed no cognitive impairment, dementia and, to a lesser extent, MCI were independently associated with a 
Fig. 1. Kaplan-Meier estimates of unadjusted 90-day survival stratified by pre-stroke cognitive status. MCI, mild cognitive impairment.

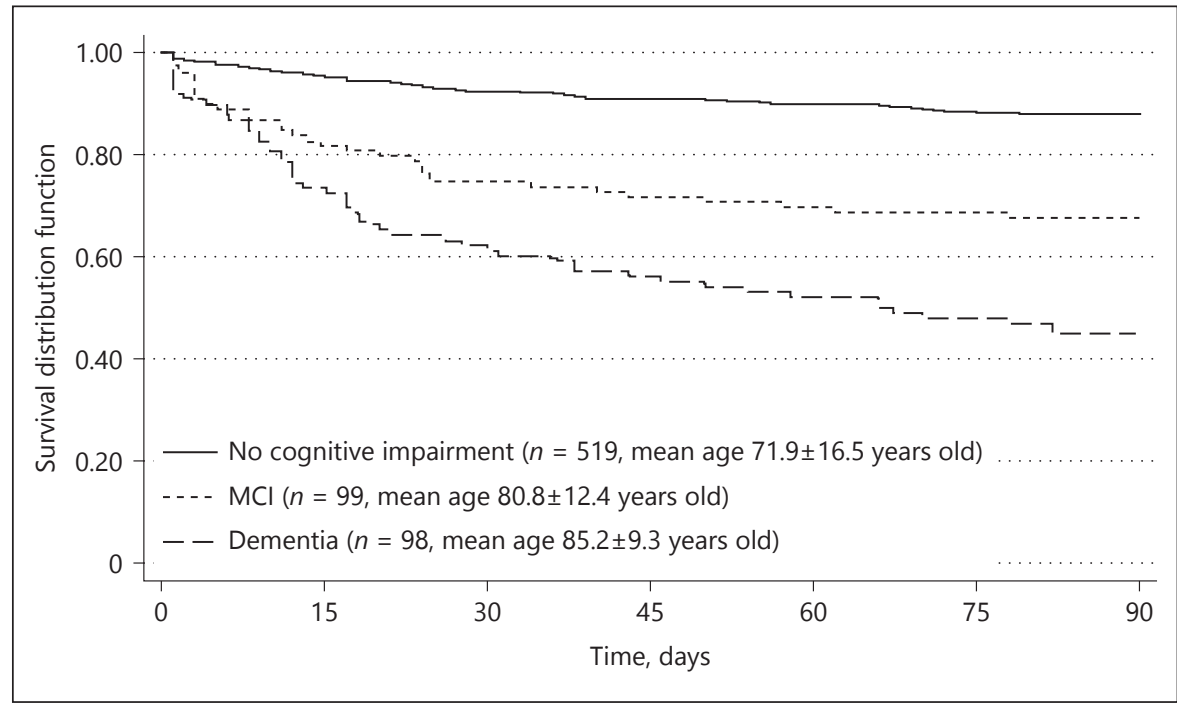

Table 2. Association between pre-stroke cognitive status and 90day case-fatality in overall patients

\begin{tabular}{|c|c|c|c|}
\hline & HR & $95 \% \mathrm{CI}$ & $p$ value \\
\hline \multicolumn{4}{|l|}{ Unadjusted } \\
\hline No cognitive impairment & Ref. & & \\
\hline MCI & 3.15 & $2.06-4.84$ & $<0.001$ \\
\hline Dementia & 6.16 & $4.26-8.89$ & $<0.001$ \\
\hline \multicolumn{4}{|l|}{ Model $1^{*}$} \\
\hline No cognitive impairment & Ref. & & \\
\hline MCI & 2.44 & $1.58-3.77$ & $<0.001$ \\
\hline Dementia & 4.08 & $2.77-6.01$ & $<0.001$ \\
\hline \multicolumn{4}{|l|}{ Model $2^{\$}$} \\
\hline No cognitive impairment & Ref. & & \\
\hline MCI & 1.90 & $1.13-3.17$ & 0.014 \\
\hline Dementia & 3.14 & $1.96-5.05$ & $<0.001$ \\
\hline \multicolumn{4}{|l|}{ Model $3^{\S}$} \\
\hline No cognitive impairment & Ref. & & \\
\hline MCI & 2.22 & $1.21-4.05$ & 0.009 \\
\hline Dementia & 4.35 & $2.49-7.61$ & $<0.001$ \\
\hline $\begin{array}{l}* \text { Adjusted for age and sex. } \\
\$ \text { Model } 1 \text { plus hypertensio } \\
\text { ing status, coronary heart dise } \\
\text { ure, active cancer, previous str } \\
\S \text { Model } 2 \text { plus pre-stroke } \\
\text { MCI, mild cognitive impa } \\
\text { of Health Stroke Scale. }\end{array}$ & $\begin{array}{l}\text { diabe } \\
\text { e, alcol } \\
\text { ke, str } \\
\text { pende } \\
\text { ment; }\end{array}$ & $\begin{array}{l}\text { atrial fibril } \\
\text { l consumpti } \\
\text { e type, and I } \\
\text { y. } \\
\text { IHSS, Natio }\end{array}$ & $\begin{array}{l}\text { tion, smok- } \\
\text { theart fail- } \\
\text { IHSS score. } \\
\text { al Institutes }\end{array}$ \\
\hline
\end{tabular}

greater risk of death at 90 days. In-hospital complications did not differ between groups, except for more frequent acute confusion in those with MCI and dementia.

Limited data about cognitive status before stroke are available in the literature. In a meta-analysis, only 3 pop- ulation-based studies that assessed pre-stroke dementia were identified, and the prevalence of the disease was 9.1\% [6]. More recently, a prevalence of pre-stroke dementia of $8 \%$ was reported in the OXVASC study [17]. This study included patients with TIA, which could have contributed to the lower prevalence as compared with our findings. In hospital-based studies, the prevalence was estimated to be greater (14.4\%) [6]. Information about MCI before stroke is scarce, and, to the best of our knowledge, no other population-based study has reported such data. In a hospital-based cohort, $12.6 \%$ of stroke patients had a cognitive impairment without dementia before the event [18]. Another study restricted to IS patients treated with IV thrombolysis identified $31 \%$ of patients with pre-existing MCI, but patients with prior dementia were excluded [19]. In a multicenter study, also limited to IS patients who received thrombolysis therapy, pre-stroke cognitive impairment (including both dementia and MCI) accounted for $30 \%$ of the cohort [20]. In these studies, the Informant Questionnaire of Cognitive Decline in the Elderly (IQCODE) was used to determine the presence of pre-stroke cognitive impairment.

Previous studies have reported that pre-existing dementia may be associated with a greater risk of death after stroke $[7-9,21]$. There are several explanations for this observation, including the fact that patients with dementia are older and tend to have more severe strokes and more comorbidities, as observed in our results. In line with our findings, it has been reported that patients with dementia tend to be less frequently admitted to a stroke unit, and less frequently treated with IV thrombolysis [10]. In our analyses, these variables were adjusted to ac- 
count for differences in baseline characteristics and management of patients, including pre-stroke dependency, and yet the excess in post-stroke mortality was still observed, suggesting the influence of other factors. Among these, it has been suggested that hospitalized stroke patients with dementia could be at greater risk of acute medical complications [8-10]. However, we did not observe any difference in the prevalence of post-stroke complications according to cognitive status, except for a higher frequency of acute confusion in patients with either dementia or MCI. Previous studies have also found that preexisting cognitive decline is associated with post-stroke delirium [22], thus contributing to higher mortality [23]. However, acute confusion alone cannot explain the excess in mortality in patients with dementia or MCI since it was still observed after adjustment for this variable in Cox models, and it is likely that certain unmeasured confounders play a deleterious role on post-stroke prognosis. Patients with dementia are frailer than their non-dementia counterparts, and it can be assumed that frailty may increase the vulnerability to adverse health outcomes, especially in stroke patients. Since frailty was not formally assessed in our study, we cannot exclude that this variable could account for the greater risk of death in patients with dementia. In line with this hypothesis, a recent study demonstrated that frailty, and especially its physical domains including slow walking speed and grip strength, were associated with a shorter survival and a poorer outcome in terms of activities of daily living in stroke patients aged 65-99 years [24].

Another consideration is the medical approach to patients with dementia. The practical approach in the management between these patients and non-demented patients may differ. It has been shown that stroke patients with dementia less frequently undergo certain diagnostic procedures $[10,25]$, swallowing assessments [10], or invasive treatments such as endotracheal intubation [26]. This may result from an assumption that both the application and the benefit of these types of procedures are limited for patients with dementia, especially those considered in poor health.

The new finding of the present study is that beyond dementia, pre-existing MCI was also associated with higher post-stroke mortality. Direct comparison with the literature is difficult since the impact of prior MCI on stroke outcome has been poorly investigated. Contrary to our results, 3-month mortality did not differ in patients with cognitive impairment, no-dementia and patients with normal cognition in 1 small study ( $n=99$ patients) restricted to IS treated with IV thrombolysis [19]. Several
Table 3. Association between pre-stroke cognitive status and 90day case-fatality in hospitalized patients only

\begin{tabular}{lccc}
\hline & HR & 95\% CI & $p$ value \\
\hline $\begin{array}{l}\text { Unadjusted } \\
\quad \text { No cognitive impairment }\end{array}$ & Ref. & & \\
$\quad$ MCI & 3.24 & $2.11-4.99$ & $<0.001$ \\
$\quad$ Dementia & 6.24 & $4.22-9.24$ & $<0.001$ \\
Model 1 & & & \\
$\quad$ No cognitive impairment & Ref. & & \\
$\quad$ MCI & 2.53 & $1.63-3.92$ & $<0.001$ \\
$\quad$ Dementia & 4.23 & $2.81-6.37$ & $<0.001$ \\
Model $2^{\$}$ & & & \\
$\quad$ No cognitive impairment & Ref. & & \\
$\quad$ MCI & 2.03 & $1.22-3.40$ & 0.007 \\
$\quad$ Dementia & 3.35 & $2.04-5.49$ & $<0.001$ \\
Model 3 & & & \\
$\quad$ No cognitive impairment & Ref. & & \\
$\quad$ MCI & 1.91 & $1.13-3.21$ & 0.015 \\
$\quad$ Dementia & 2.97 & $1.79-4.91$ & $<0.001$ \\
Model $4^{\text {a }}$ & & & \\
$\quad$ No cognitive impairment & Ref. & & \\
$\quad$ MCI & 2.25 & $1.22-4.16$ & 0.01 \\
$\quad$ Dementia & 4.08 & $2.24-7.41$ & $<0.001$ \\
\hline
\end{tabular}

* Adjusted for age and sex.

\$ Model 1 plus hypertension, diabetes, atrial fibrillation, smoking status, coronary heart disease, heart failure, active cancer, previous stroke, stroke type, and NIHSS score.

$\S$ Model 2 plus admission to a stroke care unit.

"Model 3 plus pre-stroke dependency.

MCI, mild cognitive impairment; NIHSS, National Institutes of Health Stroke Scale.

Table 4. In-hospital medical complications and length of stay of stroke patients according to pre-stroke cognitive status

\begin{tabular}{|c|c|c|c|c|}
\hline & $\begin{array}{l}\text { No cognitive } \\
\text { impairment } \\
(n=499)\end{array}$ & $\begin{array}{l}\text { MCI } \\
(n=96)\end{array}$ & $\begin{array}{l}\text { Dementia } \\
(n=78)\end{array}$ & $p$ value \\
\hline $\begin{array}{l}\text { Length of stay in days, } \\
\text { median (IQR) }\end{array}$ & $10(5-18)$ & $12(5-18)$ & $9(2-20)$ & 0.46 \\
\hline Recurrent stroke & 2.4 & 0 & 3.9 & 0.20 \\
\hline Hemorrhagic & & & & \\
\hline transformation* & 5.8 & 4.0 & 3.5 & 0.65 \\
\hline Systemic bleeding & 2.4 & 3.1 & 1.3 & 0.73 \\
\hline Infection & 24.5 & 29.2 & 32.1 & 0.27 \\
\hline Venous thromboembolism & 4.0 & 2.1 & 5.1 & 0.55 \\
\hline Arterial ischemic & & & & \\
\hline complication & 1.8 & 4.2 & 1.3 & 0.29 \\
\hline Acute confusion & 2.8 & 16.7 & 10.3 & $<0.001$ \\
\hline Epileptic seizure & 2.4 & 3.1 & 2.6 & 0.92 \\
\hline Other complications & 10.4 & 16.7 & 9.0 & 0.17 \\
\hline
\end{tabular}


explanations could account for the greater mortality observed in patients with pre-existing MCI. Stroke occurrence may accelerate cognitive deterioration, as suggested by several studies that reported pre-stroke cognitive impairment as a risk factor for post-stroke dementia [21, 27]. In addition, as in demented patients, frailty may worsen post-stroke prognosis in MCI patients.

The main strength of our study is the populationbased design that avoids a baseline selection bias related to hospital-based methodology [17] and consequently ensures more reliable estimations. Conversely, because of this design, it was not possible to administrate formal scales such as the IQCODE to assess pre-stroke cognitive status. Instead, we proceeded to interview patients, their relatives or their general practitioner, as well as to review the medical files to determine pre-existing MCI and dementia. Hence, we cannot exclude that some patients with cognitive impairment were classified as having MCI instead of dementia because of an underestimation of the actual interference of cognitive impairment on daily activities. However, the fact that the prevalence of pre-existing MCI and dementia was consistent with previous reports indicate that this limitation should have limited impact. In addition, despite rigorous investigations, information about pre-stroke cognitive status was missing in $6 \%$ of patients. In these patients (median age: 88 years old [interquartile range 79-91], 63\% women), 3-month case-fatality rate was $54 \%$, close to that observed in demented patients. Of note, these patients did not differ with either patients with dementia, or those with MCI, with regard to stroke type, NIHSS score, or vascular risk factors (data not shown). In contrast, stroke severity was greater than that of patients with normal cognition (median NIHSS score 12, interquartile range 3-20, $p<0.001$ ); they had a greater prevalence of atrial fibrillation $(40.5 \%$, $p=0.007)$ and heart failure $(14.3 \%, p=0.005)$, and a low- er prevalence of current smoking $(9.5 \%, p=0.025)$. Therefore, it could be assumed that a substantial proportion of these patients could have in fact pre-stroke cognitive impairment. This would be in favor of an underestimation of the true effect of dementia and MCI on the risk of death, thus reinforcing our key findings. Finally, medical complications in hospitalized patients were assessed only during the acute portion of the stay. Therefore, we cannot conclude that not-hospitalized patients with dementia or MCI had more frequent acute complications that contributed to mortality when considering the overall cohort. However, when analyses were restricted to hospitalized patients, the excess in mortality in MCI patients and patients with dementia was still observed.

To conclude, pre-existing MCI and dementia were both associated with increased 90 -day mortality in stroke patients. Such associations were not fully explained by baseline characteristics, stroke severity or patient management, and need to be explored by further studies to identify the underlying mechanisms.

\section{Acknowledgments}

None.

\section{Disclosure Statement}

Y.B. received honoraria for or consulting fees from AstraZeneca, Daiichi-Sankyo, Bayer, BMS, Pfizer, Novex Pharma, Medtronic, MSD France, Amgen, and Boehringer-Ingelheim. Other authors: none.

\section{Funding Sources}

None.

\section{References}

1 Feigin VL, Lawes CM, Bennett DA, BarkerCollo SL, Parag V. Worldwide stroke incidence and early case fatality reported in 56 population-based studies: a systematic review. Lancet Neurol. 2009 Apr;8(4):355-69.

2 Feigin VL, Forouzanfar MH, Krishnamurthi R, Mensah GA, Connor M, Bennett DA, et al.; Global Burden of Diseases, Injuries, and Risk Factors Study 2010 (GBD 2010) and the GBD Stroke Experts Group. Global and regional burden of stroke during 1990-2010: findings from the Global Burden of Disease Study 2010. Lancet. 2014 Jan;383(9913):24554.
3 Krishnamurthi RV, Feigin VL, Forouzanfar $\mathrm{MH}$, Mensah GA, Connor M, Bennett DA, et al.; Global Burden of Diseases, Injuries, Risk Factors Study 2010 (GBD 2010); GBD Stroke Experts Group. Global and regional burden of first-ever ischaemic and haemorrhagic stroke during 1990-2010: findings from the Global Burden of Disease Study 2010. Lancet Glob Health. 2013 Nov;1(5): e259-81.

4 Béjot Y, Bailly H, Durier J, Giroud M. Epidemiology of stroke in Europe and trends for the 21st century. Presse Med. 2016 Dec;45(12 Pt 2):e391-8.
5 Wu YT, Fratiglioni L, Matthews FE, Lobo A Breteler MM, Skoog I, et al. Dementia in western Europe: epidemiological evidence and implications for policy making. Lancet Neurol. 2016 Jan;15(1):116-24.

6 Pendlebury ST, Rothwell PM. Prevalence, incidence, and factors associated with prestroke and post-stroke dementia: a systematic review and meta-analysis. Lancet Neurol. 2009 Nov;8(11):1006-18.

7 Barba R, Morin MD, Cemillán C, Delgado C, Domingo J, Del Ser T. Previous and incident dementia as risk factors for mortality in stroke patients. Stroke. 2002 Aug;33(8):1993-8. 
8 Hénon H, Durieu I, Lebert F, Pasquier F, Leys D. Influence of prestroke dementia on early and delayed mortality in stroke patients. J Neurol. 2003 Jan;250(1):10-6.

9 Appelros P, Viitanen M. What causes increased stroke mortality in patients with prestroke dementia? Cerebrovasc Dis. 2005; 19(5):323-7.

10 Saposnik G, Cote R, Rochon PA, Mamdani M, Liu Y, Raptis S, et al.; Registry of the Canadian Stroke Network; Stroke Outcome Research Canada (SORCan) Working Group. Care and outcomes in patients with ischemic stroke with and without preexisting dementia. Neurology. 2011 Nov;77(18):1664-73.

11 Subic A, Cermakova P, Norrving B, Winblad B, von Euler M, Kramberger MG, et al. Management of acute ischaemic stroke in patients with dementia. J Intern Med. 2017 Apr; 281(4):348-64.

12 Sudlow CL, Warlow CP. Comparing stroke incidence worldwide: what makes studies comparable? Stroke. 1996 Mar;27(3): $550-8$.

13 Bennett DA, Brayne C, Feigin VL, BarkerCollo S, Brainin M, Davis D, et al. Development of the Standards of Reporting of Neurological Disorders (STROND) checklist: A guideline for the reporting of incidence and prevalence studies in neuroepidemiology. Neurology. 2015 Sep;85(9):821-8.

14 Lainay C, Benzenine E, Durier J, Daubail B, Giroud M, Quantin C, et al. Hospitalization within the first year after stroke: the Dijon stroke registry. Stroke. 2015 Jan;46(1):1906.

15 WHO. The world health report 2000: Health Systems improving performance. Geneva: WHO; 2000.

16 Williams LS, Yilmaz EY, Lopez-Yunez AM. Retrospective assessment of initial stroke severity with the NIH Stroke Scale. Stroke. 2000 Apr;31(4):858-62.

17 Pendlebury ST, Chen PJ, Bull L, Silver L, Mehta Z, Rothwell PM; Oxford Vascular Study. Methodological factors in determining rates of dementia in transient ischemic attack and stroke: (I) impact of baseline selection bias. Stroke. 2015 Mar;46(3):641-6.

18 Serrano S, Domingo J, Rodríguez-Garcia E, Castro MD, del Ser T. Frequency of cognitive impairment without dementia in patients with stroke: a two-year follow-up study. Stroke. 2007 Jan;38(1):105-10.

19 Murao K, Bodenant M, Cordonnier C, Bombois S, Hénon H, Pasquier F, et al. Does preexisting cognitive impairment no-dementia influence the outcome of patients treated by intravenous thrombolysis for cerebral ischaemia? J Neurol Neurosurg Psychiatry. 2013 Dec;84(12):1412-4.

20 Murao K, Leys D, Jacquin A, Kitazono T, Bor$\operatorname{det} \mathrm{R}$, Béjot Y, et al.; OPHELIE-COG investigators. Thrombolytic therapy for stroke in patients with preexisting cognitive impairment. Neurology. 2014 Jun;82(23):2048-54.
21 Desmond DW, Moroney JT, Sano M, Stern Y. Mortality in patients with dementia after ischemic stroke. Neurology. 2002 Aug;59(4):53743.

22 Oldenbeuving AW, de Kort PL, Jansen BP, Roks G, Kappelle LJ. Delirium in acute stroke: a review. Int J Stroke. 2007 Nov;2(4): 270-5.

23 Shi Q, Presutti R, Selchen D, Saposnik G. Delirium in acute stroke: a systematic review and meta-analysis. Stroke. 2012 Mar;43(3): 645-9.

24 Winovich DT, Longstreth WT Jr, Arnold AM, Varadhan R, Zeki Al Hazzouri A, Cushman $M$, et al. Factors Associated With Ischemic Stroke Survival and Recovery in Older Adults. Stroke. 2017 Jul;48(7):1818-26.

25 Béjot $Y$, Jacquin A, Troisgros O, Rouaud O, Aboa-Eboulé C, Hervieu M, et al. Diagnostic procedures in ischaemic stroke patients with dementia. a population-based study. Int J Stroke. 2015 Jan;10(1):95-8.

26 Busl KM, Nogueira RG, Yoo AJ, Hirsch JA, Schwamm LH, Rost NS. Prestroke dementia is associated with poor outcomes after reperfusion therapy among elderly stroke patients. J Stroke Cerebrovasc Dis. 2013 Aug;22(6): 718-24.

27 Hénon H, Durieu I, Guerouaou D, Lebert F, Pasquier F, Leys D. Poststroke dementia: incidence and relationship to prestroke cognitive decline. Neurology. 2001 Oct;57(7): 1216-22. 